This is the author's version of:

Blanca Ramírez Barat, Emilio Cano, "The use of agar gelled electrolyte for in situ electrochemical measurements on metallic cultural heritage" Electrochimica Acta, Volume 182, 2015, Pages 751-762, ISSN 0013-4686,

https://doi.org/10.1016/j.electacta.2015.09.116. 


\title{
The use of agar gelled electrolyte for in situ electrochemical measurements on metallic cultural heritage
}

\author{
Blanca Ramírez Barat and Emilio Cano \\ Centro Nacional de Investigaciones Metalúrgicas (CENIM), Consejo Superior de Investigaciones \\ Científicas (CSIC), Av. Gregorio del Amo 8, 28040 Madrid (Spain) \\ blanca.ramirez@csic.es, ecano@cenim.csic.es
}

\begin{abstract}
:
Electrochemical techniques, such as electrochemical impedance spectroscopy (EIS), are widely used for corrosion studies. However, their applicability to studies on metallic cultural heritage has been less spread due to the practical difficulties of performing measurements in-situ on sculptures or monuments. One interesting approach to this application is the use of gel polymer electrolytes (GP-E) to overcome the difficulties of handling liquid electrolytes on irregular leaning surfaces. In this paper, the behavior of an agar gelled electrolyte with a portable cell is evaluated for EIS measurements over three types of bronze coupons, and compared with a traditional liquid cell. The influence of the addition of agar in the reproducibility and repeatability of measurements is assessed, as well as the possible interaction of agar with the corrosion process. Results show that, although agar slightly accelerates the anodic process, it does not significantly affect the cathodic reaction and does not introduce new reactions in the corrosion mechanism. It is demonstrated that the GP-E allows obtaining reproducible and good quality EIS spectra, comparable to the liquid cell. Hence, it can be used for comparative in-situ measurements, being a very valuable tool for the evaluation of patina and coatings on metal cultural heritage.
\end{abstract}

Keywords: agar; polymer electrolyte; cultural heritage; EIS; bronze.

\section{Introduction}

Electrochemical Impedance Spectroscopy (EIS) is a widely used technique for corrosion studies, as it gives qualitative and quantitative information on corrosion processes and corrosion resistance. In the field of metallic cultural heritage it can be a very useful tool as it can be used to evaluate the actual situation of an object, i.e., if there is an active corrosion process or it has a stable patina, how much a protective coating has increased its corrosion resistance, which coating offers the better corrosion protection or when a protection treatment is beginning to fail [1]. The answer to these questions can help to take conservation decisions and stablish priorities when time and resources are limited. For this reason, EIS constitutes a very valuable complement to classical analytical methods widely used in conservation science (XRF, Raman, 
XRD, FTIR, etc.), which give information on composition and structure, but cannot give quantitative information on their corrosion behavior.

However, the application of this technique for corrosion studies in the field of cultural heritage poses some particular difficulties [1]. Some studies have been carried out using artificial coupons that try to mimic the original composition of metallic artifacts [2-5], or patinas scrapped from the monuments [6], using traditional laboratory techniques. Nevertheless, this kind of laboratory studies provide limited information, due to the impossibility to reproduce the composition and characteristics of patinas that have formed over several hundred years. On the other hand, it is always desirable to evaluate conservation conditions on the real objects to undertake conservation decisions. At this point, the main issue is how to perform electrochemical measurements using a conventional three electrode cell with a liquid electrolyte on a non-flat, irregular and leaning surface as is usually the one of metallic sculptures and monuments.

Since the beginning of application of electrochemical techniques to cultural heritage, mainly electrochemical impedance spectroscopy (EIS), researchers have worked on portable devices with different approaches. On one side, methods for retaining the liquid electrode in contact with the object have been developed, as the Letardi's contact probe [7, 8]. This method has been successfully applied to the evaluation of cultural heritage, but still has de disadvantage of handling a liquid electrolyte. On the other side, commercial gel electrodes or prepared gel electrodes based on same type of gels have also been proposed $[9,10]$. This system avoids the liquid electrolyte, so it is convenient for field use. Nevertheless, the conductivity of these gels is poor, so irregular distribution of currents might mislead the results [11]. Additionally, the composition of the electrolyte cannot be selected to test the resistance of the metal to specific environments in commercial electrodes, or it is limited by the swelling equilibrium of synthetized (anionic) gels [12]. This is a major drawback of this approach, since for corrosion studies the composition and $\mathrm{pH}$ of the electrolyte play a key role in the process. So although the idea of gel electrolytes seems to be a good choice, solutions that allow the selection of the adequate electrolyte have to be further investigated.

The use of solid electrolytes has been explored in the last decades for application where liquid electrolytes present inconveniences such as handling difficulties or risk of leaking or evaporation of the liquid [13]. The development of new and improved solid electrolytes has mainly focused in the fields of energy storage (lithium batteries, solar and fuel cells...) and electrochromic devices. Different kinds of polymers have been assayed as solid electrolytes, from polyethylene oxide (PEO) since the 70's of the last century to polymer mixtures, composites or hybrid inorganic-organic polymer electrolytes. In last years, natural polymers have been considered as a cheap and green alternative, including gelatin, chitosan, agar, etc. Among these, agar offers interesting properties making it a good candidate for these applications.

Agar is a natural polysaccharide extracted from certain species of red seaweeds. It is composed of two fractions, agarose and agaropectin, both made up of repeating units of agarobiose. 
While agarose is a neutral linear polymer, agaropectin is partially modified by different hydrophobic (methoxyl) and polar (sulfate, pyruvate) side groups. [14]

Since its discovery agar has been using as a gelling agent for many different uses. In this case it has been the choice as it is inexpensive, easy and quick to prepare and can support a wide range of aqueous electrolytes, forming translucent gels. Furthermore, agar has a very interesting property, syneresis, which is the ability of weeping or expelling liquid from a gel [15].This helps wetting the surface and favors contact between the electrolyte and the working electrode (i.e., the metal under study) in field corrosion tests.

Previous studies have been carried out on agar based electrolytes, but these studies have focused on its preparation with different salts or acids in order to achieve the maximum conductivity, with good optical and mechanical properties [16-18]. For its use as electrolyte in electrochemical corrosion measurements, our interest is focused on its use with classical aqueous electrolytes usually employed to evaluate the behavior of one metal in different weathering conditions. In the case of evaluation of cultural heritage objects, several electrolytes have been proposed to simulate marine, urban or rural atmospheres. Nevertheless, the use of these electrolytes may damage the original surface of the object so authors working in this field have proposed mild electrolytes, included slightly mineralized water to avoid introducing aggressive ions on the patinas [7].

Taking this into account, and as an alternative to previously mentioned approaches, authors have recently developed a portable cell based on the use of agar as a gelling agent to support the liquid electrolyte [19]. While initial results have been promising, yielding results that are comparable to a conventional liquid cell, a deeper study of the effect of agar on the results is needed: changes in the electrolyte conductivity were observed with the addition of agar, but have not been studied; and the possible effect of agar in the corrosion process needs to be well understood before the extensive application of this cell for in-situ corrosion measurements.

Therefore, the aim of this work is to evaluate the behavior of an agar-gelled electrolyte for its use in a portable cell for in situ electrochemical corrosion measurements on cultural heritage, in order to assess the stability and reproducibility of the measurements and the possible effect of agar in the results.

\section{Experimental}

The design of the gel polymer electrolyte (GP-E) cell is described in a previous paper in detail [19], based on a traditional three electrode cell as shown in figure 1, were WE is the working electrode or the object under study, CE is the counter made with a AISI 316L stainless steel mesh and RE is a $99.9 \%$ silver wire (Goodfellow) electrochemically coated with $\mathrm{AgCl}$, used as (pseudo) reference electrode [20]. 

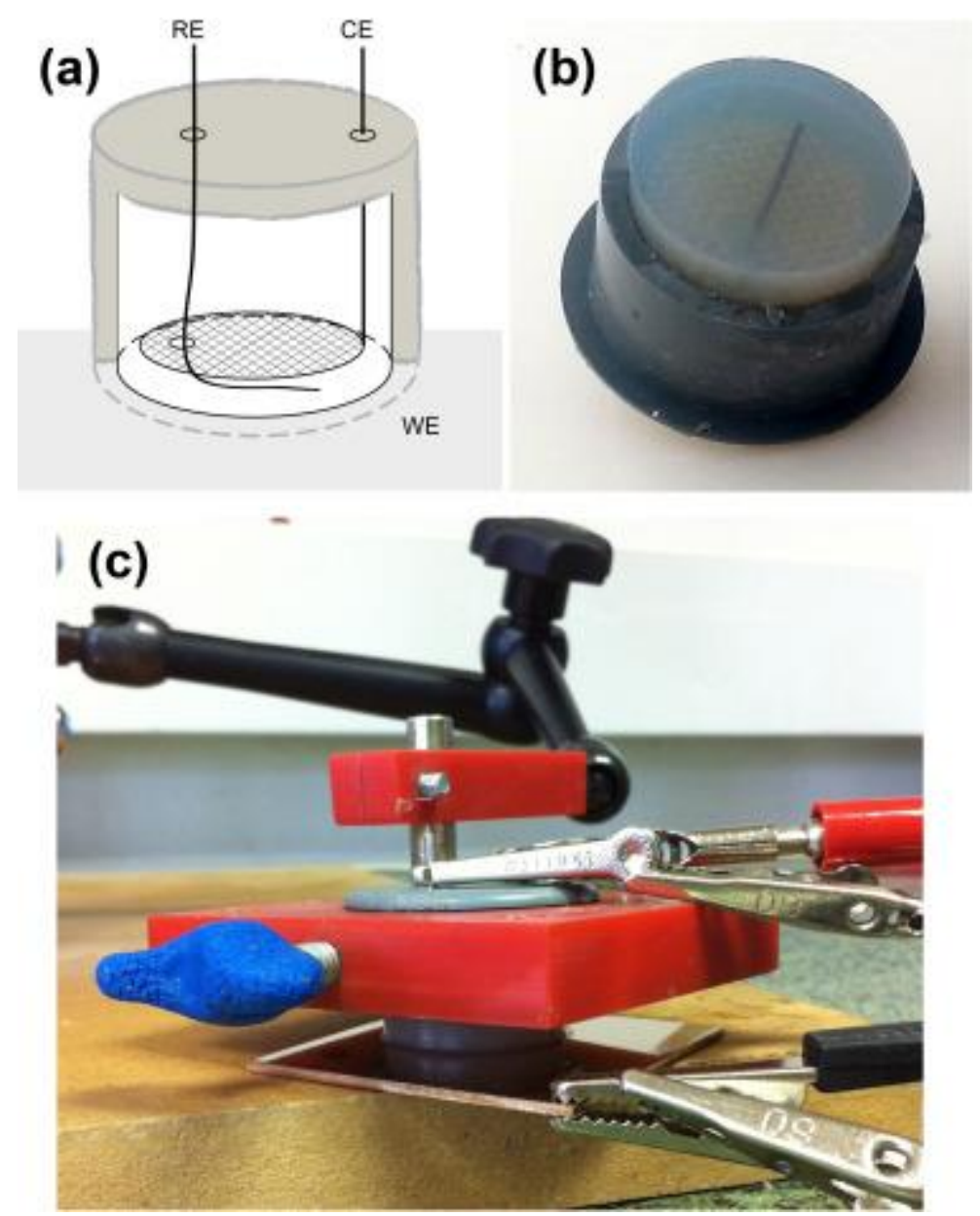

Fig. 1. Cell design scheme (a), picture of the cell with the gelled electrolyte from the side in contact with the WE (b) and picture of the cell/sample connection setup (c).

The electrolyte has been prepared by gelling artificial rain with agar (technical grade). To minimize the introduction of aggressive ions and to mimic the conditions outdoor sculptures are exposed to, the selected liquid electrolyte has been artificial rain adapted from [21]. Since the conductivity of this solution is too low, it has been used ten times concentrated. The composition of the electrolyte is presented in Table 1 . The chloride concentration in this solution is $3.58 \cdot 10^{-4} \mathrm{M}$. This gives a potential of $0,462 \mathrm{~V}$ vs. SHE for the $\mathrm{Ag} / \mathrm{AgCl}$ coated silver wire used as reference electrode, calculated from Nernst equation.

After the preparation of the liquid electrolyte, 1 to $5 \% \mathrm{w} / \mathrm{v}$ of agar powder has been dispersed in the solution, heated until dissolution, allowed to cool down for a few minutes and casted on the electrochemical cell containing the RE and CE. For comparison, the same electrochemical cell and electrode arrangement has also been used with the liquid electrolyte without agar (agar $0 \%$ ). The addition of agar slightly increased the $\mathrm{pH}$ of the electrolyte, from a value of 6.6 measured in the liquid solution to a 7.0 value in the $5 \%$ agar electrolyte. 
Table 1. Composition of the $10 x$ concentrated synthetic rain used as electrolyte.

\begin{tabular}{lc}
\hline Composition & Conc. mg/l \\
\hline $\mathrm{CaSO}_{4} \cdot 2 \mathrm{H}_{2} \mathrm{O}$ & 14.43 \\
$\left(\mathrm{NH}_{4}\right)_{2} \mathrm{SO}_{4}$ & 15.04 \\
$\left(\mathrm{NH}_{4}\right) \mathrm{Cl}$ & 19.15 \\
$\mathrm{NaNO}_{3}$ & 15.13 \\
$\mathrm{CH}_{3} \mathrm{COONa}$ & 3.19 \\
\hline $\mathrm{pH}$ adjusted to 6.5 with $\mathrm{HNO}_{3}$ & \\
\hline
\end{tabular}

Electrochemical measurements have been carried out on two types of samples: $50 \times 50 \times 1.5 \mathrm{~mm}$ laminated EN 1652 CuSn5 bronze coupons ( $95 \mathrm{Cu}, 5 \mathrm{Sn} \% \mathrm{w}$ ), hereafter bronze A; and $70 \times 35 \times 7$ mm EN 1982 CC491K (DIN 1705-RG5) cast bronze coupons (85 Cu, $5 \mathrm{Sn}, 5 \mathrm{~Pb}, 5 \mathrm{Zn} \mathrm{\% w),}$ hereafter bronze $B$. Bronze $A$ was selected as a simple model to minimize other variables in the study of the agar behavior, while bronze $B$ was selected to have a system as close as possible to real cast bronze sculptures.

Bronze A was used as received to have a long-term natural oxide layer. Bronze B was prepared to mimic bronze sculptures by Codina Escultura, a traditional Spanish artistic foundry according to traditional materials and techniques: Bronze was casted in ingots, cut into coupons of the desired size and sandblasted. Then, two different artificial patinas have been applied to bronze $B$, a dark potassium sulfide patina and a green ammonium chloride patina, following traditional patination procedures for artistic sculpture. For the dark patina, a $10 \% \mathrm{w} / \mathrm{v}$ aqueous solution of potassium sulfide was applied by brush and then heated with a blowtorch. Green patina was obtained with a $10 \% \mathrm{w} / \mathrm{v}$ ammonium chloride aqueous solution applied with brush over the dark one. Figure 2 shows the aspect of the coupons.

EIS spectra have been acquired using a Gamry 600 Potentiostat, using a frequency swept from $100 \mathrm{kHz}$ to $10 \mathrm{mHz}, 10 \mathrm{mV}$ RMS amplitude (at the open circuit potential, OCP) and 10 points/decade. The area exposed to the electrolyte was $3 \mathrm{~cm}^{2}$. Analysis of the data has been carried out using ZView software. Polarization curves have been obtained starting from -150 $\mathrm{mV}$ vs. OCP and polarizing at $0.16 \mathrm{mV} / \mathrm{s}$ in the anodic direction.

The system was left to stabilize at OCP for 30 minutes before measurements. As it has been observed and confirmed by other authors [22], the most remarkable variations show in the 
first 30 minutes and stay small after that time. Between different measurements 5-10 minutes delay has shown to be enough for the stabilization of the OCP.

Conductivity and $\mathrm{pH}$ of the electrolytes has been measured with a Crison MM40 conductimeter/pHmeter.

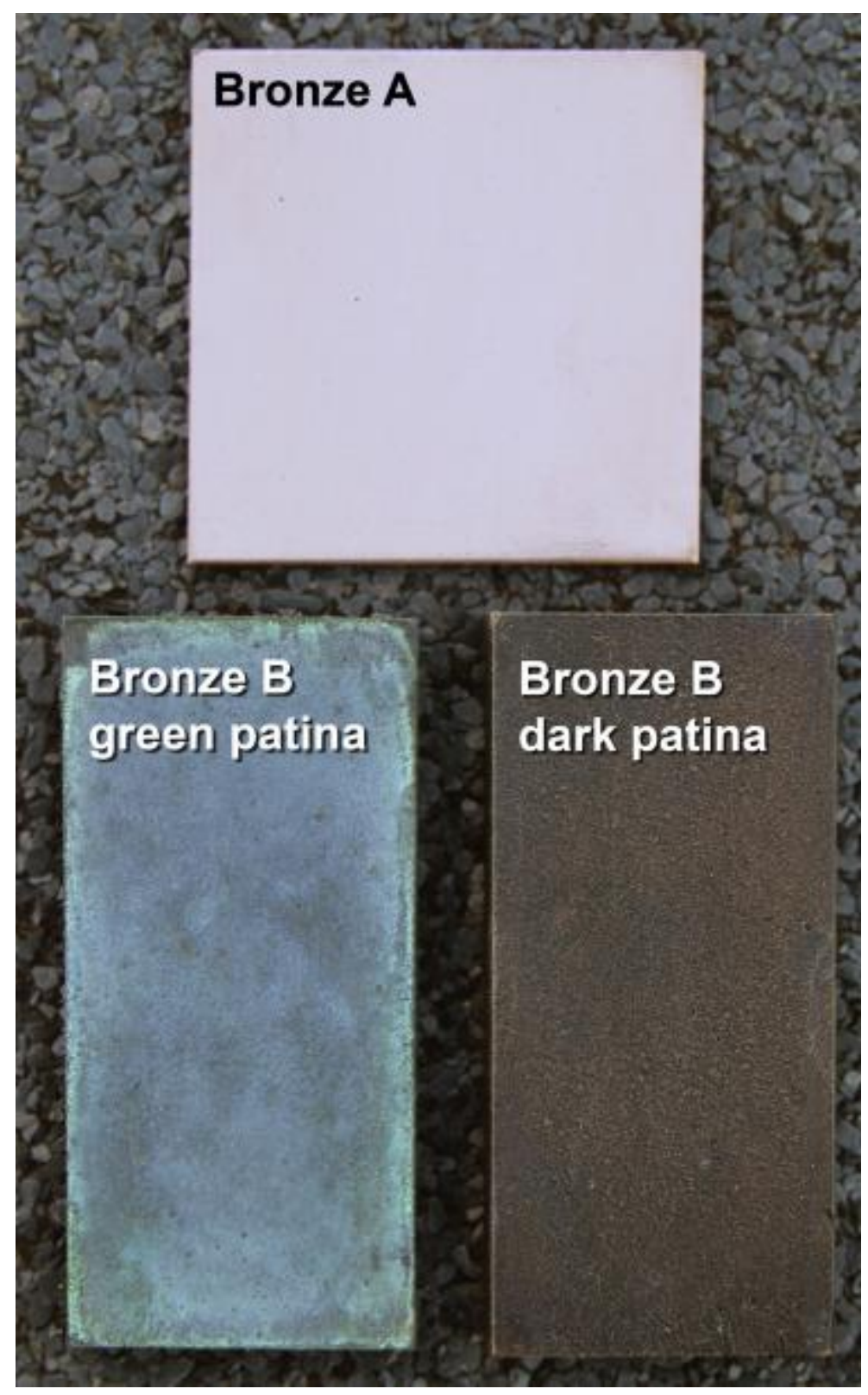

Fig. 2. Bronze coupons used for the electrochemical tests: clean bronze $A$ (top), and artificially patinated bronze $B$, with a dark potassium sulfide patina (bottom right) and a green ammonium chloride patina (bottom left).

\section{Results and discussion}

In order to validate the system and verify its stability three consecutive EIS measurements were performed on the same sample. Although the system takes some time to stabilize 7 
undergoing small changes with time, the general repeatability of the measurements is quite good. Figure 3 shows an example of three consecutive measurements on a bronze A coupon. Only small differences in the mid frequency appear, being related to slight modifications of surface oxide layers in contact with the electrolyte. Kramers-Kronig analysis was applied to all curves demonstrating the linearity, stability, causality and finite value of all measurements. Results for the patina-covered bronze B samples were even more stable. The small changes in the spectra also demonstrate that the measurements do not alter the surface of the bronze, i.e., the tests are non-destructive and can be safely applied to cultural heritage objects.
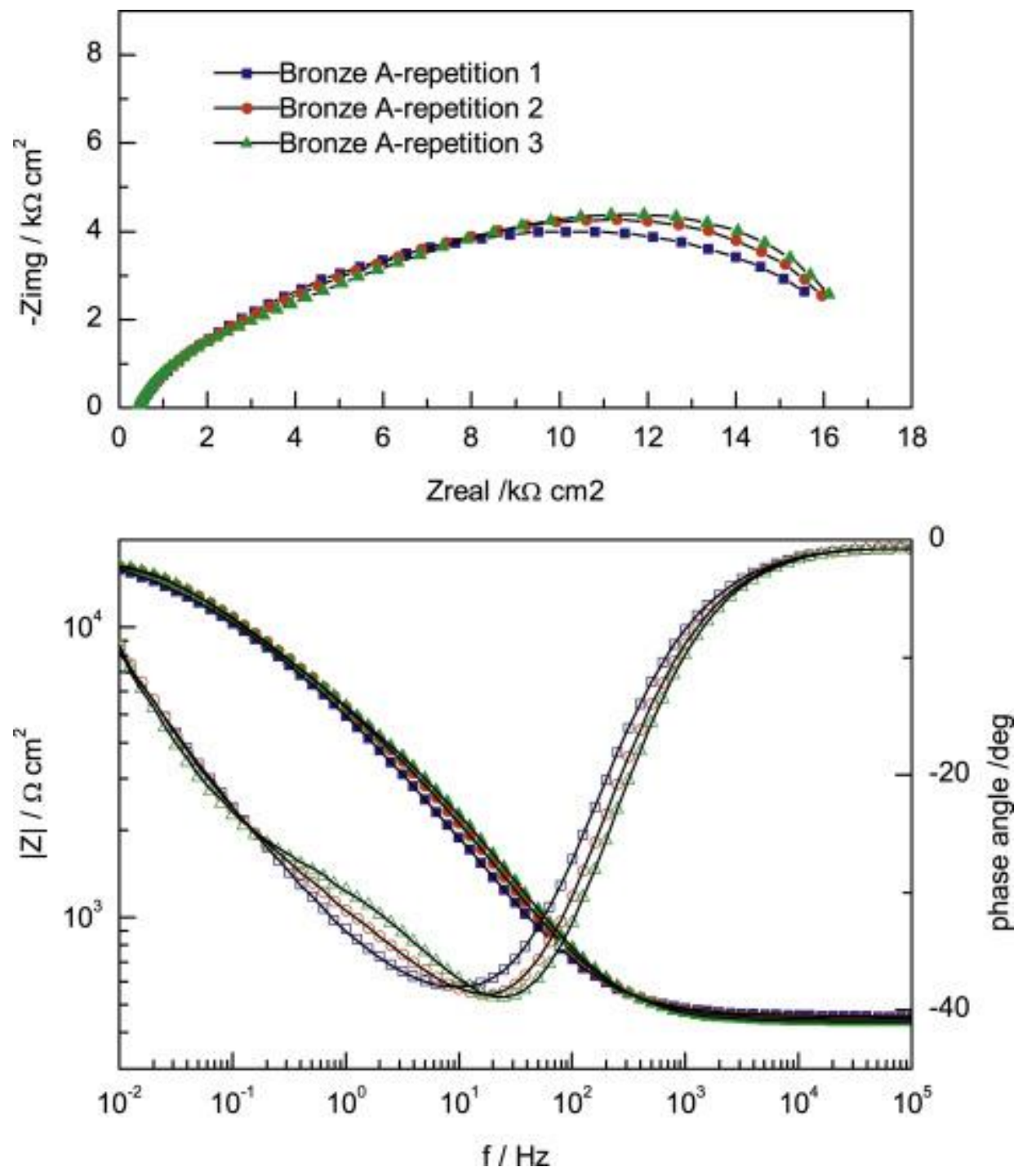

Fig. 3. Nyquist (top) and Bode (bottom) plots showing three consecutive measurements on a bronze $A$ coupon. GP-E cell with 3\% agar electrolyte. Black lines represent the Kramers-Kronig fitting of data.

Repeatability was also checked in different areas of the same sample. In figure 4 two independent measurements on a bronze B coupon covered with dark patina show that measurements on similar surfaces give almost identical responses. 

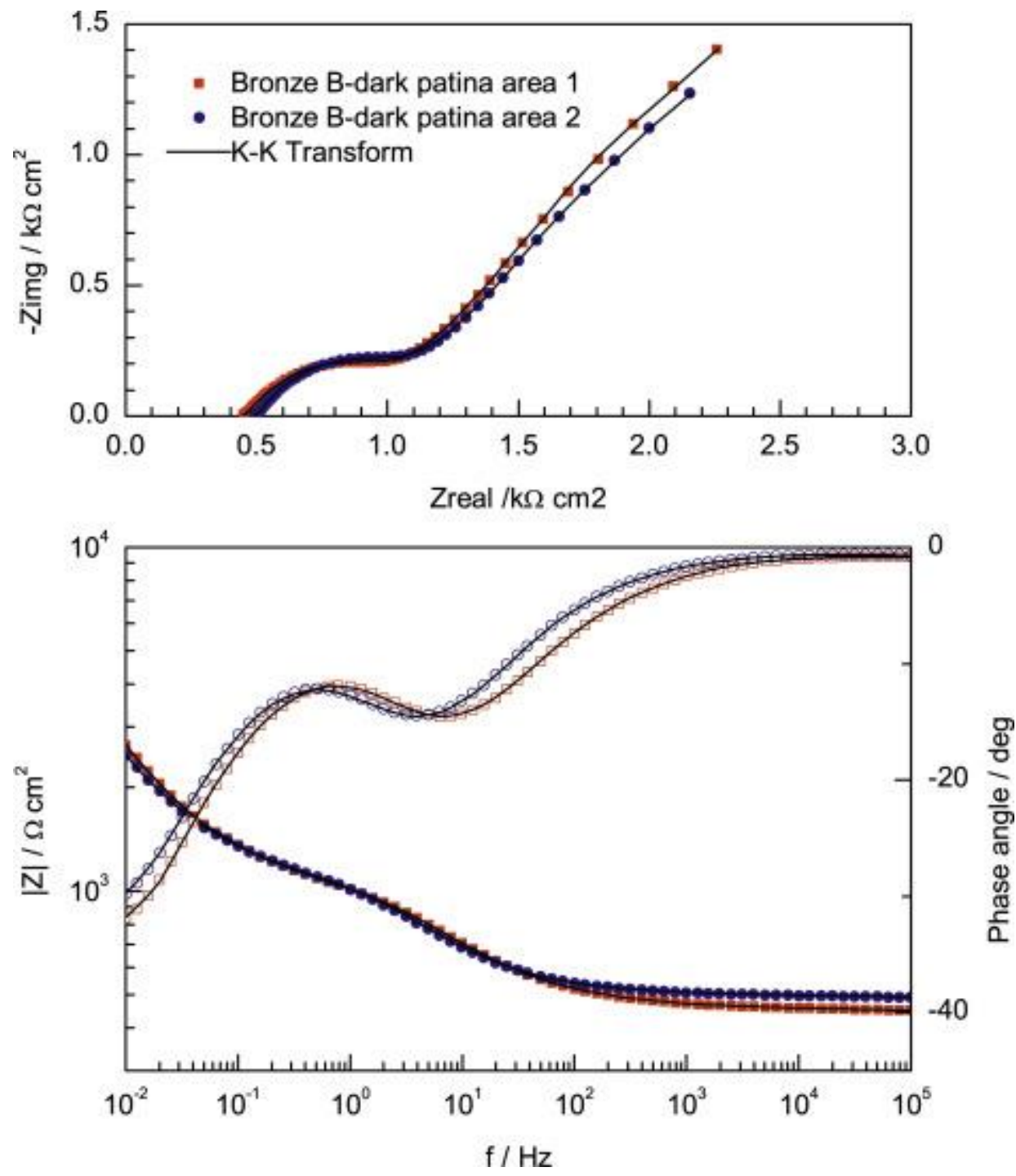

Fig. 4. Nyquist (top) and Bode (bottom) plots of two different areas from a bronze B coupon with dark patina. GP-E cell with 3\% agar electrolyte. Black line represents the Kramers-Kronig fitting of data.

The possible effects of agar was evaluated comparing spectra registered with the same cell filled with liquid electrolyte and gelled electrolyte ( $3 \%$ agar) on different samples. Results are shown in figures 5 (Bronze A), 6 (Bronze B, dark patina) and 7 (Bronze B, green patina). The outline from both curves is comparable, the same features being present, although the addition of agar results in a decrease of $Z$ module and a shift of time constants to higher frequencies. As $|\mathrm{Z}|$ at high frequencies is related with electrolyte resistance, this indicates that agar addition increases the electrolyte conductivity. The decrease of $|Z|$ at low frequencies and the frequency shift of time constants suggest a direct effect of agar in the corrosion process. 

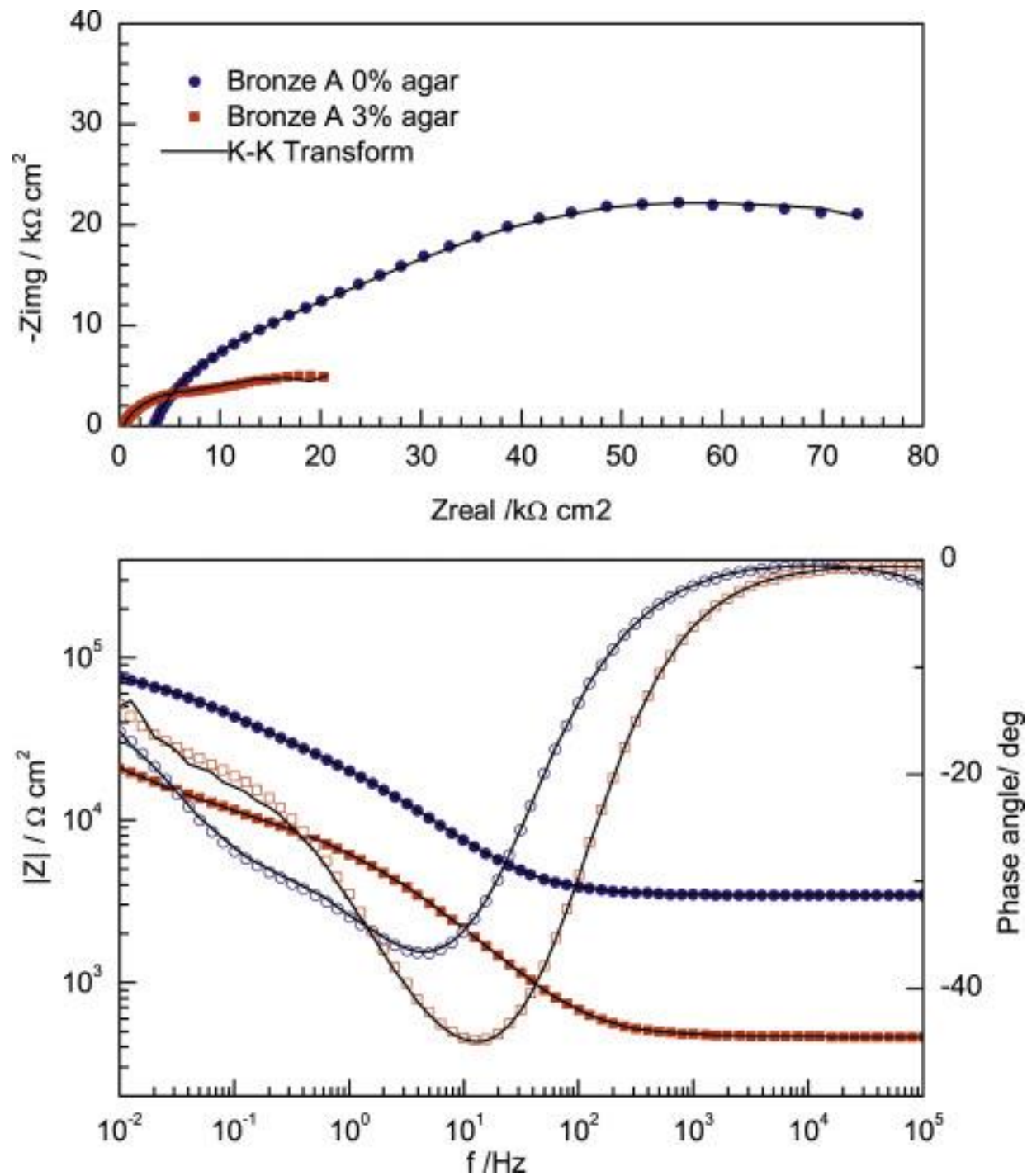

Fig. 5. Nyquist (top) and Bode (bottom) plots of a bronze A coupon. GP-E cell with $3 \%$ agar electrolyte (squares) and same cell with liquid electrolyte (circles). Black line represents the Kramers-Kronig fitting of data. 

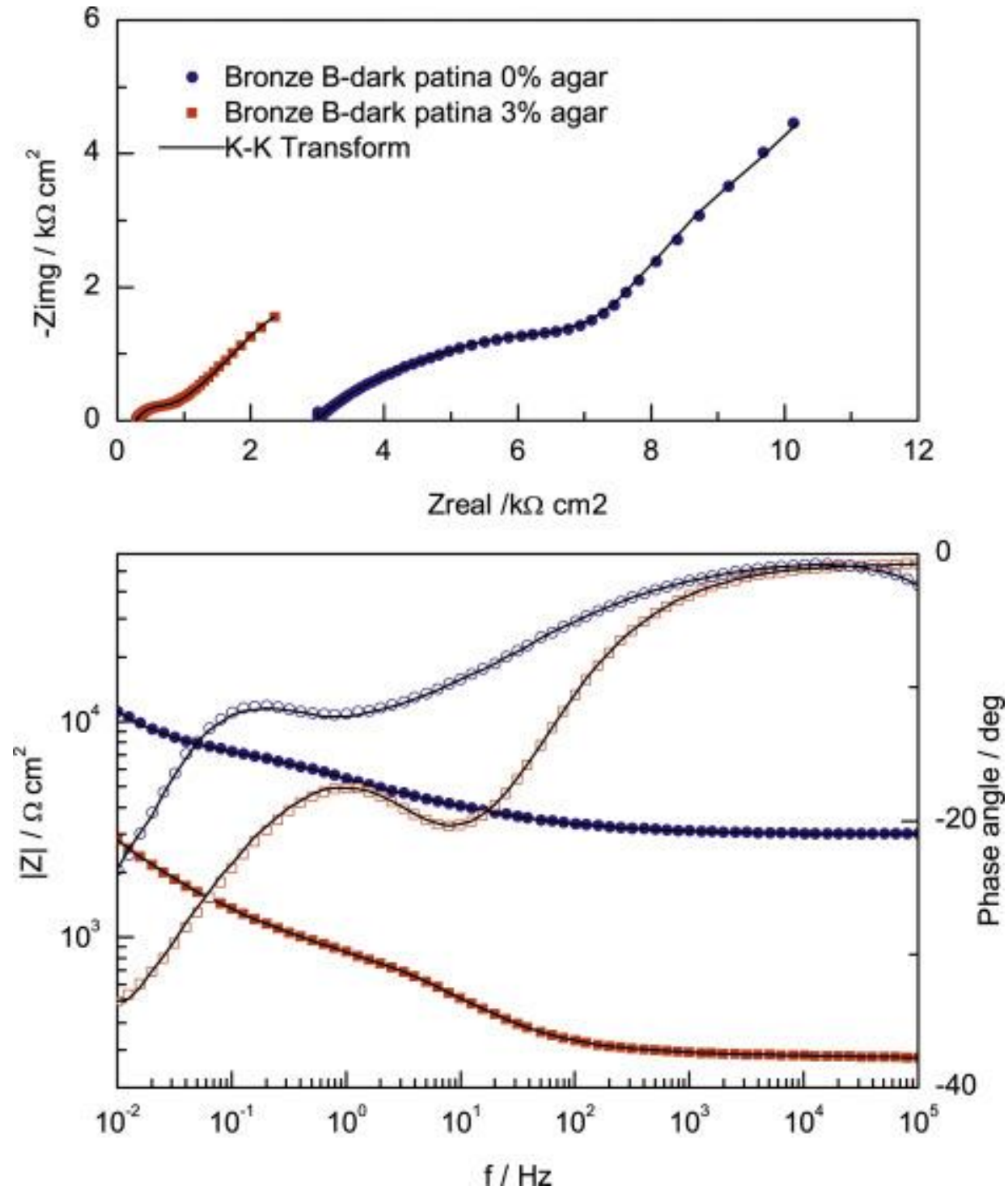

Fig. 6. Nyquist (top) and Bode (bottom) plots of a bronze B coupon with dark patina. GP-E cell with $3 \%$ agar electrolyte (squares) and same cell with liquid electrolyte (circles). Black line represents the Kramers-Kronig fitting of data. 

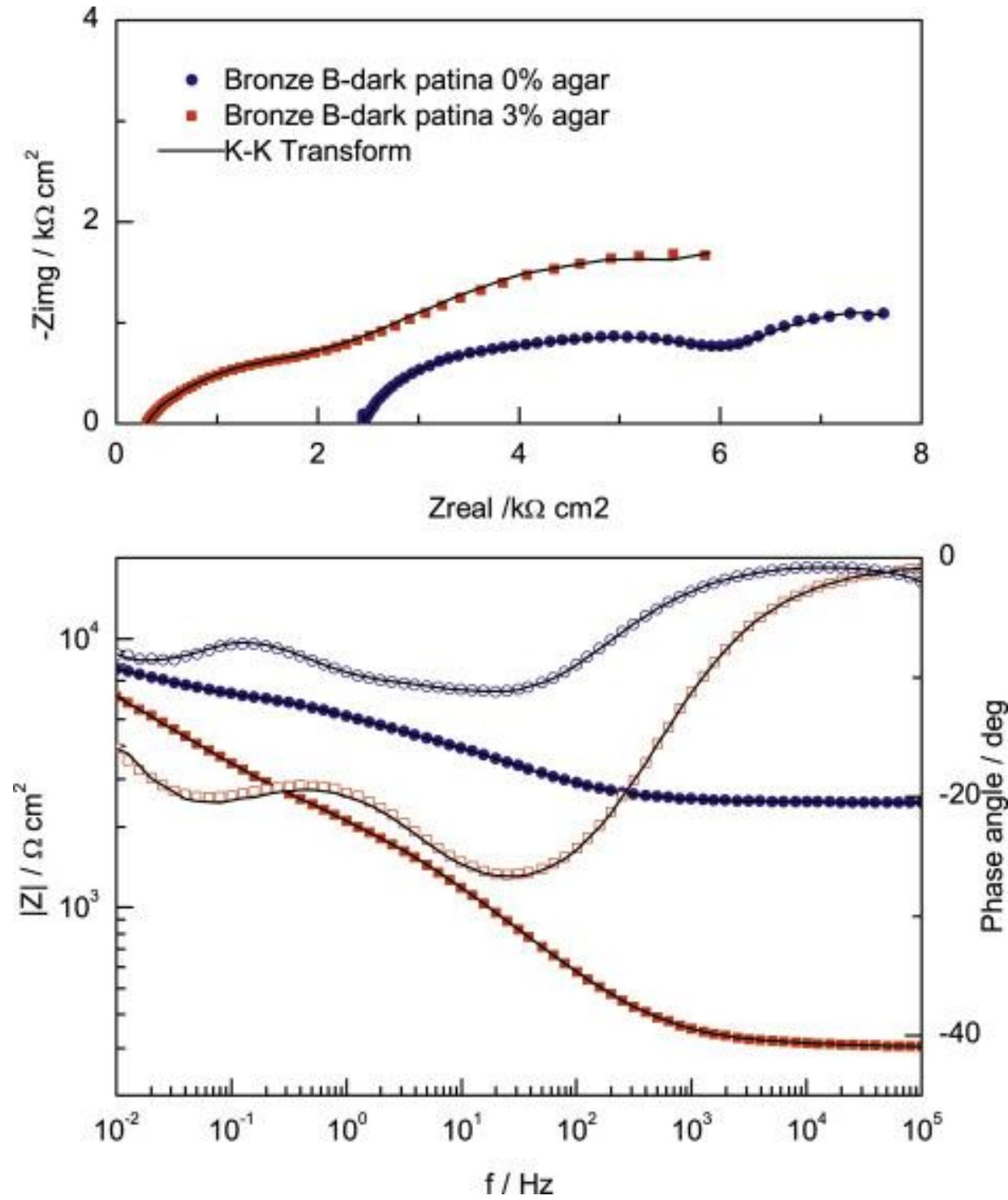

Fig. 7. Nyquist (top) and Bode (bottom) plots of a bronze B coupon with green patina. GP-E cell with $3 \%$ agar electrolyte (squares) and same cell with liquid electrolyte (circles). Black line represents the Kramers-Kronig fitting of data.

In order to obtain a better understanding of agar influence in our system, different agar concentrations, from 0 to $5 \%$ were assayed, using bronze $A$ as a simple model to eliminate the effect of possible inhomogeneities of the different patinas. Agar addition might influence two processes: the resistance of the electrolyte and the corrosion reaction on the metal/electrolyte interface. For our system to be valid to carry out in situ measurements on metallic heritage, corrosion mechanisms should not be significantly altered by the addition of agar. The electrolyte conductivity, on the other hand, is a parameter that can be substracted from experimental EIS data, so it does not interfere on the interpretation of the results. 


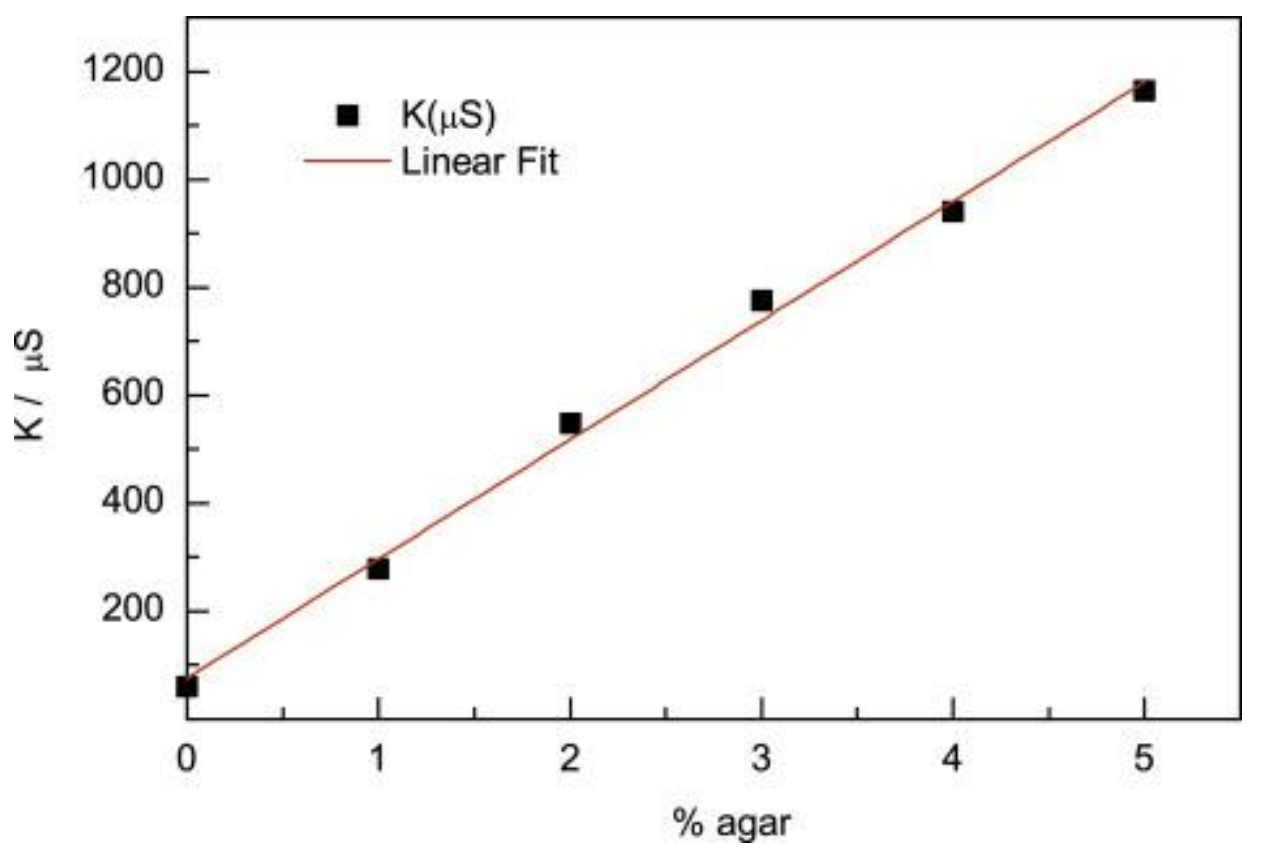

Fig. 8. Conductivity increase of the electrolyte with agar concentration.

To see how resistance of the electrolyte changes with agar, conductivity of electrolyte with different agar concentrations (0-5\%) was measured (Figure 8). It can be observed that electrolyte conductivity increases proportionally to agar concentration, being a linear function of the concentration of agar plus the conductivity of the electrolyte, according to:

$$
\mathrm{K}=76+221 \text { [\%agar] } \quad \mathrm{R}^{2}=0.99
$$

An additional measurement was done with 3\% agar in deionized water to separate the contribution of the liquid electrolyte (artificial rain), giving a value of $720 \pm 10 \mu \mathrm{S}$. So, gelled electrolyte's conductivity is the sum of two contributions: agar's conductivity and artificial rain's conductivity, being agar the main responsible of the electrolyte's conductivity. This may represent an advantage for its application in cultural heritage, as it gives higher conductivity to the electrolyte without introducing other ions that may leave dangerous residues on the surface of the object.

Aiming to understand the corrosion processes, several electrochemical measurements including EIS and polarization curves have been done for 0 to $5 \%$ agar. Polarization curves have been registered to elucidate the effect of the agar on the anodic and cathodic reactions. Figure 9 show the polarization curves obtained in bronze A. Under our experimental conditions (aerated neutral pH electrolyte), the expected main anodic and cathodic reactions are:

$$
\text { Anodic reaction: } \mathrm{Cu}^{0} \leftrightarrow \mathrm{Cu}^{+}+1 \mathrm{e}^{-}
$$

Cathodic reaction: $2 \mathrm{H}_{2} \mathrm{O}+\mathrm{O}_{2}+4 \mathrm{e}^{-} \leftrightarrow 4 \mathrm{OH}^{-}$(3) 
The cathodic branch seems to follow a Tafel behavior with no significant changes in the slopes with the increasing concentration of agar. This indicates that the cathodic process (eq. 3 ) is under charge transfer control and that the addition of agar up to $5 \%$ does not substantially change the kinetics of this reaction. On the other hand, agar clearly affects the anodic branch, increasing the anodic slopes as the concentration of agar increases, and suggests a direct interaction between agar and copper ions which shifts the anodic reaction towards the oxidation of copper (eq. 2).

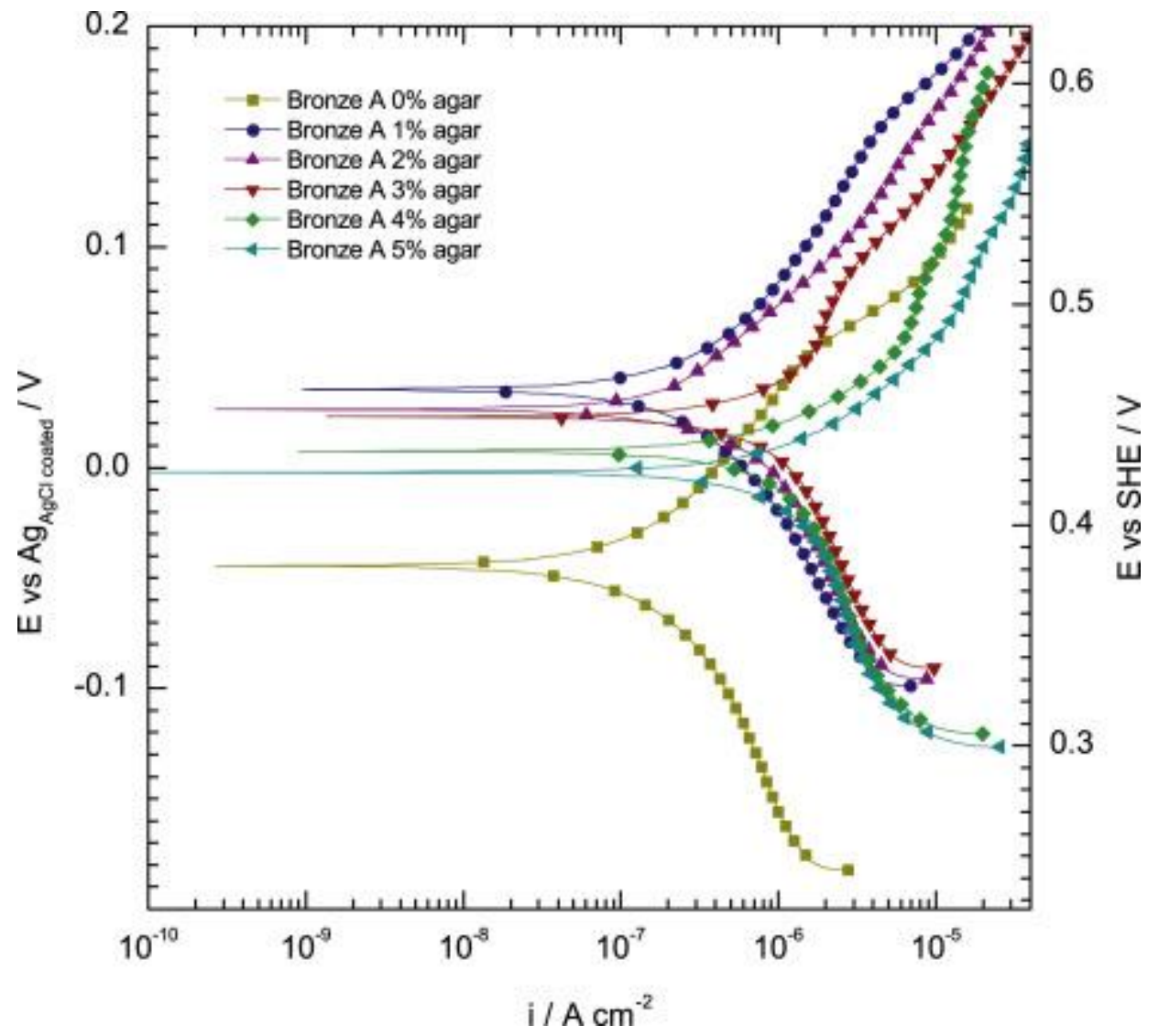

Fig. 9. Polarization curves obtained in bronze A with different agar concentration.

Experimental EIS data for 0-5\% agar electrolytes are presented in figure 10. The general shape of the spectra shows two time constants. The increase of electrolyte conductivity whit agar concentration is clearly appreciated in the impedance at high frequencies. Also, a decrease in the size of the capacitive loops in Nyquist plots is observed with increase in agar concentration. 

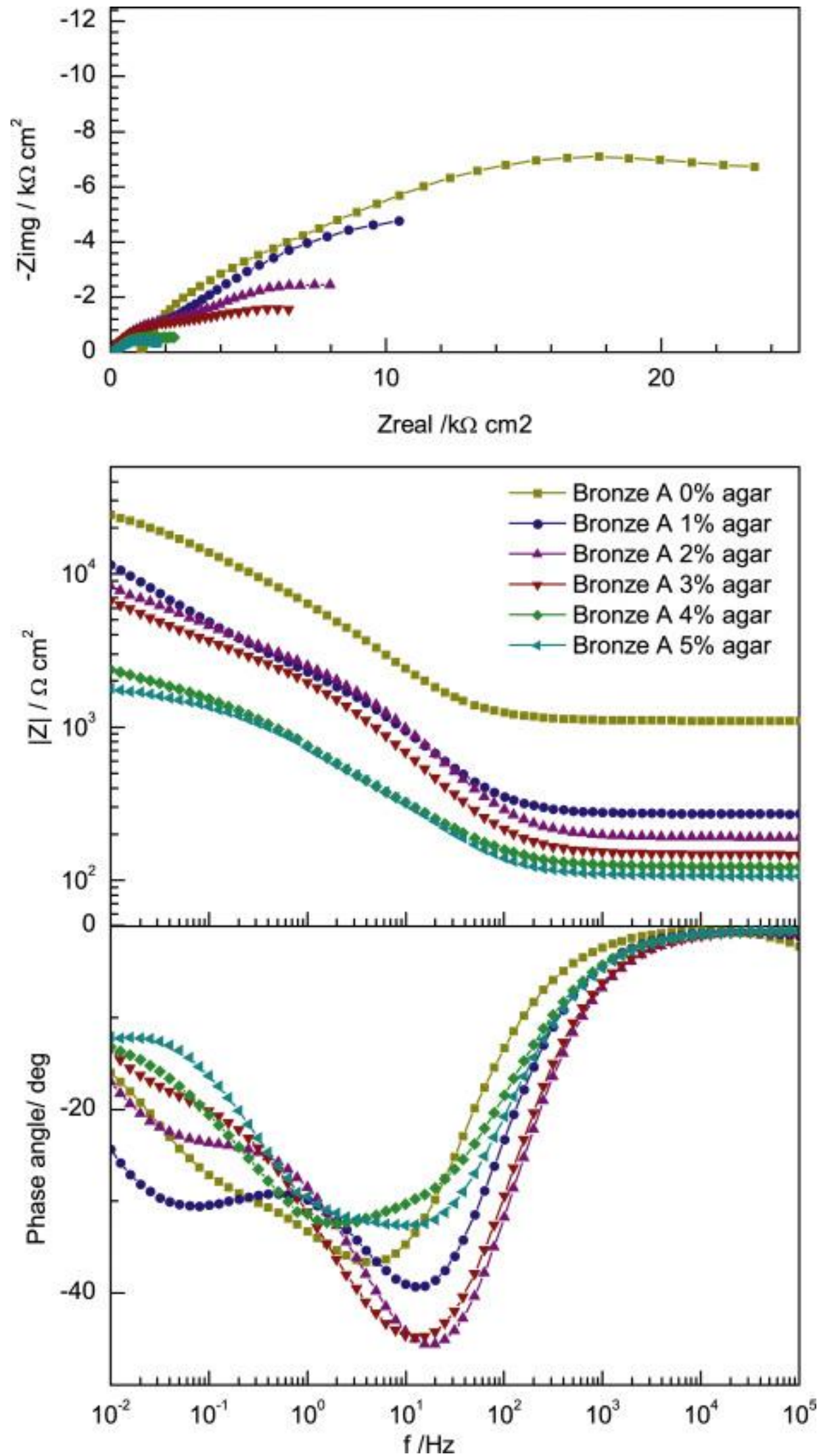

Fig. 10. Nyquist (top) and Bode (bottom) plots of EIS data for bronze A with different concentrations of agar $0-5 \%$. 
The large changes in the electrolyte resistance $\left(R_{0}\right)$ obscure the analysis of the effect of agar in the corrosion reactions on the metal-electrolyte interface. To avoid this effect, electrolyte resistance (obtained from intersection of the extrapolation of high frequency loop with the real axis in Nyquist plots) has been substracted from the EIS data. This representation eliminates the distortion induced by this resistance and allows [23]. Additionally, data above $10^{4} \mathrm{~Hz}$ showed artifacts attributable to instrumental effects, and therefore have been eliminated for analysis. Corrected ( $\left.Z-R_{0}\right)$ impedance modulus versus frequency data are presented in figure 11 . The features of the curves for all concentrations of agar are similar, indicating that the addition of agar does not alter significantly the electrode reactions, thus supporting the use of this gelled electrolyte for EIS measurements.

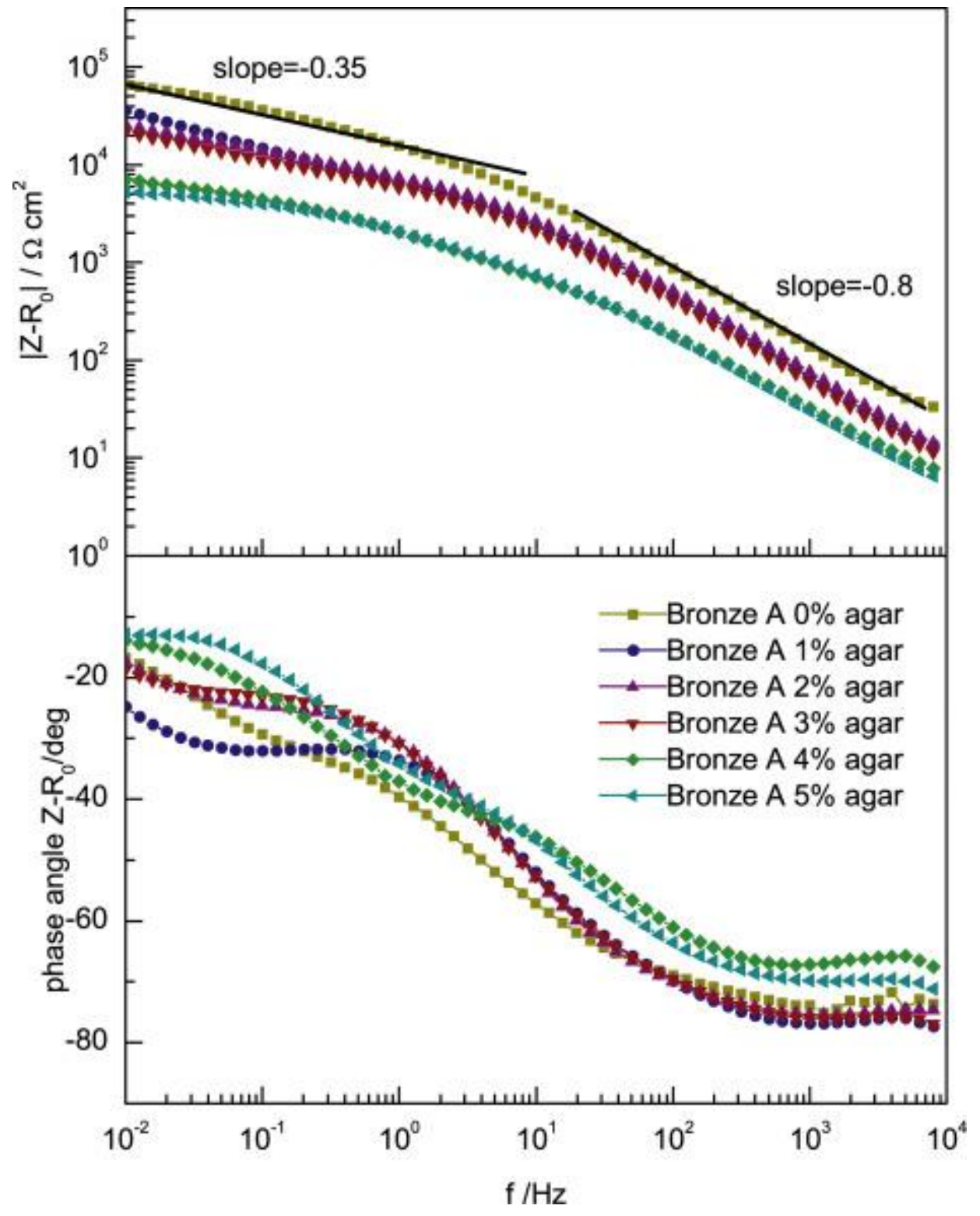

Fig. 11. Modulus (top) and phase angle (bottom) of corrected impedance (Z-R0) as function of frequency for bronze A with different concentrations of agar 0-5\%. 
Two different slopes can be clearly observed in figure 11. The slope of the high frequency part of the data (above $10 \mathrm{~Hz}$ ) gives a value of $\sim-0.8$ for all measurements, and the low frequency part (below $1 \mathrm{~Hz}$ ) has a slope between 0.3 and 0.35 . These slopes depart from the ideal capacitive behavior (slope of -1 ), indicating a distribution of the time constants. This distribution of the time constants is commonly modeled by a constant phase element (CPE):

$$
Z_{C P E}=\frac{1}{Y(j \omega)^{\alpha}}
$$

whose actual physical meaning could be very different depending on the system: this response has been attributed to surface roughness and heterogeneities, to electrode porosity, to variation of coating composition, to slow adsorption reactions, and to non-uniform potential or current distributions [24].

As a first approach experimental data were fitted to an equivalent circuit with 2 nested CPE- $R$ pairs, as shown in figure 12 a. To give a physical meaning to the mathematical analysis several possibilities may be evaluated.

(a)

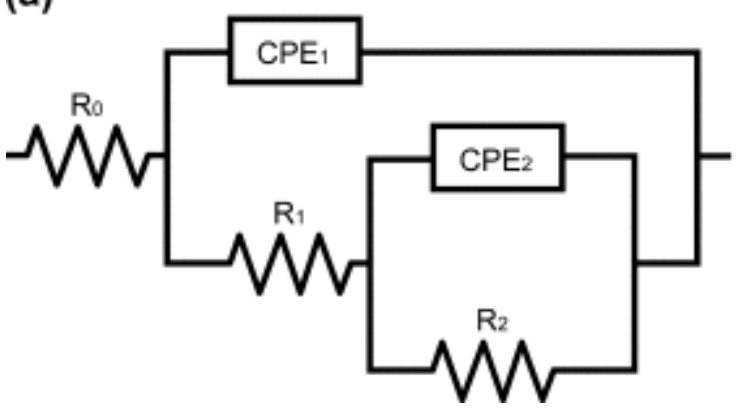

(b)

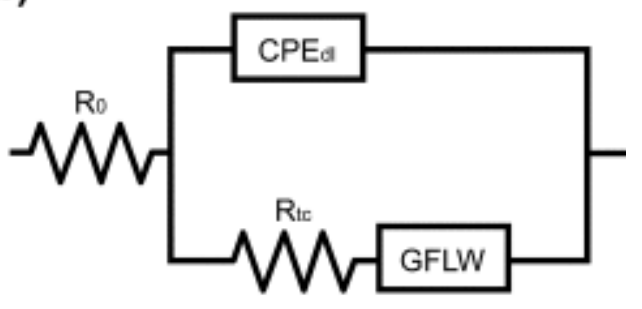

Fig. 12. Equivalent electrical circuits used to analyze EIS data.

One approach it to interpret the results according to the classical equivalent circuit for modeling an imperfect metal-coating system, in which elements of circuit 12a are interpreted as: $\mathrm{R}_{0}$ represents the electrolyte resistance, $\mathrm{CPE}_{1}$ represents the pseudo-capacitance of the patina or oxide layer on the metal surface and $R_{1}$ the resistance of the electrolyte though the pores; and $\mathrm{CPE}_{2}$ in parallel with $\mathrm{R}_{2}$ represent the pseudo-capacitance of the double layer at the metal-electrolyte interface and the charge transfer resistance of the corrosion reaction respectively. In this hypothesis the distribution of time constants of $\mathrm{CPE}_{2}$ would be related to inhomogeneous current distributions due to both irregularities in the metal surface [20] and to the presence of agar molecules at the interface. As agar molecules interact with the metal surface and favor the corrosion process, an increase of agar would explain the decrease of impedance contribution associated to $\mathrm{CPE}_{2}$.

Nevertheless, the values of $\alpha_{2}$ with this model are about 0.5 for all conditions. This value is too low to be attributed to inhomogeneities or roughness of the surface, especially considering the smooth and apparently regular surface of bronze A (see Fig. 2), that is was not altered by the 
measurements. Recent works have also demonstrated that pure geometric aspects (surface roughness) can not explain a CPE behavior [25, 26].

A second tentative interpretation of the distribution of the time constant at low frequencies is to assign it to the impedance of a porous electrode. Under de Levie's assumptions (equal cylindrical pores uniformly distributed in the electrode surface), the impedance of a porous electrode is given by:

$Z_{\text {deLevie }}=\left(R_{c} Z_{0}\right)^{0.5} \operatorname{coth}\left(\frac{l_{c}}{\lambda}\right)$

being $R_{c}$ the electrolyte resistance within the pore, $Z_{0}$ the impedance of the flat electrode developed in the cylindrical pore, $I_{c}$ the pore depth and $\lambda=\left(Z_{0} / R_{c}\right)^{0.5}$ the penetration depth of the AC signal in the pore $[3,27]$. Hernandez et al. [3] found this kind of response on rough copper electrodes when exposed to $\mathrm{NaCl}$. In our case, the short exposure times and the low aggressiveness of the electrolyte (the chloride concentration of our electrolyte is much lower) did not change the original smooth surfaces of the sample. Since according to eq. 5 , when the pores are shallow the response of the electrode is similar to that of a flat electrode, it is not likely that the dispersion of the time constant can be attributed to porosity.

For copper corrosion in milder electrolytes, with composition and $\mathrm{pH}$ much similar to the one used in our study, the aforementioned article by Hernandez et al. (using synthetic rain of Sao Paulo, pH5) [3] and a previous one by Feng et al. [28] (using synthetic tap water, pH 7.6) found that the dispersion of the time constants was attributable to diffusion of copper ions within the oxide layer and the electrolyte. The equivalent circuit modeling this behavior is presented in figure $12 \mathrm{~b}$ in which the second CPE has been replaced by a generalized finite length Warburg impedance:

$Z_{G F L W}=\frac{R}{(T j \omega)^{\alpha}} \tanh (T j \omega)^{\alpha}$

This expression is the solution to the one-dimensional anomalous diffusion equation subject to the absorbing boundary. When $\alpha=0.5$, this equation corresponds to the finite length Warburg impedance. The numerical data for the different parameters obtained by fitting to this equivalent circuit are presented in table 2 . It should be mentioned that for 4 and $5 \%$ agar, a third time constant seems to appear at the lowest frequencies. While it is not clearly defined as to propose an additional element in the circuit, it distorts the fitting at the last points, causing inaccuracies in the estimation of the parameters of the diffusional impedance for these conditions (values marked with asterisk in the table).

The values of $\alpha_{\mathrm{GFLW}}$ are about 0.35 (corresponding to the slope of the low frequencies part of data in figure 11), therefore the diffusional impedance in our system does not follow the pure finite Warburg diffusion. The reason for this behavior is not clear, and might be related with complex diffusion phenomena or to the overlapping of other processes, such as the impedance of the thin oxide layer on the metal surface. In any case, this behavior appears both in the liquid electrolyte and the gelified electrolyte, so it is not a consequence of the addition of agar. 
Table 2. Electrochemical parameters obtained from fitting to equivalent circuit in figure $12 \mathrm{~b}$ of EIS measurements on bronze A with liquid electrolyte and gelled electrolyte with agar 1 to $5 \%$.

\begin{tabular}{|c|c|c|c|c|c|c|}
\hline \multirow[b]{2}{*}{ Agar \% } & \multicolumn{2}{|c|}{$C P E_{d l}$} & \multirow[b]{2}{*}{$\begin{array}{c}\mathrm{R}_{\mathrm{ct}} \\
\left(\mathrm{k} \Omega \mathrm{cm}^{2}\right)\end{array}$} & \multicolumn{3}{|c|}{$Z_{G F L W}$} \\
\hline & $\begin{array}{c}Y_{d l} \\
\left(\mu S s^{\alpha_{1}} \mathrm{~cm}^{-2}\right)\end{array}$ & $\alpha_{d l}$ & & $\begin{array}{c}\mathrm{R} \\
\left(\mathrm{k} \Omega \mathrm{cm}^{2}\right)\end{array}$ & $\begin{array}{l}T \\
(s)\end{array}$ & $\alpha_{w}$ \\
\hline $0 \%$ & $6.4 \pm 2$ & $0.799 \pm 0.004$ & $8 \pm 2$ & $78 \pm 5$ & $26 \pm 6$ & $0.32 \pm 0.02$ \\
\hline $1 \%$ & $8.8 \pm 0.2$ & $0.846 \pm 0.002$ & $3.4 \pm 0.1$ & $45 \pm 1$ & $40 \pm 3$ & $0.397 \pm 0.005$ \\
\hline $2 \%$ & $8.6 \pm 0.1$ & $0.837 \pm 0.001$ & $4.0 \pm 0.1$ & $33.0 \pm 0.8$ & $57 \pm 5$ & $0.311 \pm 0.004$ \\
\hline $3 \%$ & $10.3 \pm 0.2$ & $0.845 \pm 0.002$ & $2.5 \pm 0.2$ & $31 \pm 2$ & $90 \pm 20$ & $0.294 \pm 0.007$ \\
\hline $4 \%$ & $39 \pm 1$ & $0.756 \pm 0.04$ & $0.8 \pm 0.1$ & $4.9 * \pm 0.3$ & $1.9 * \pm 0.3$ & $0.38^{*} \pm 0.02$ \\
\hline $5 \%$ & $33.08 \pm 0.8$ & $0.785 \pm 0.02$ & $0.9 \pm 0.1$ & $3.9 * \pm 0.2$ & $1.5^{*} \pm 0.3$ & $0.37 * \pm 0.01$ \\
\hline
\end{tabular}

From the analysis of these results, it can be inferred that the addition of agar slightly decreases $R_{c t}$, thus increasing the anodic dissolution of copper. An increase in $C_{d l}$ is also observed, attributable to the interaction of charged moieties of agar molecules with the electrode surface. However, this increase is negligible until 3\% agar. The affinity of agar and other biomolecules for metallic ions is not unknown. There are many studies on metal uptake by different biomaterials for environmental applications [29] and agar and algae Gelidium (which is the raw material for agar extraction) have been studied as biosorbents for copper removal in wastewater [30, 31]. These biomaterials contain functional groups which may act binding sites for metal ions, therefore acting as anodic depolarizers when used in corrosion studies. A more detailed study on binding equilibrium between agar and copper would be of interest although it is out of the scope of this work.

Our results show that the addition of agar to the artificial rain electrolyte in concentrations up to $3 \%$ do alter the corrosion mechanisms, although it increases the corrosion rate by decreasing faradaic impedance. The most evident effect of the addition of agar, the decrease in the resistance of the electrolyte, can be easily substracted from experimental data, and can be even favorable for field measurements as it increases the range of measurable impedances. When using the gelled electrolyte for the assessment of protective properties of patinas or coatings on metallic cultural heritage these effects can be disregarded provided the results are reproducible and allow obtaining data comparing the protective properties of different coatings or following their evolution with time. Although corrosion rate in a determinate electrolyte will be overestimated with the addition of agar, quantitative absolute values of corrosion rates are not usually of interest when evaluating patinas or coatings for atmospheric exposure, as in these atmospheric conditions the metal is not continuously immersed in the electrolyte. These comparative results permit choosing between different coatings or monitoring changes in corrosion resistance of a surface, thus being a powerful tool for 
establishing conservation strategies for metallic cultural heritage. This application of gelled electrolyte cell for the comparison of different patinas and coating systems is currently being tested in real conservation applications. Preliminary results have shown the applicability of the agar G-PE cell as a non-destructive diagnostic tool [32, 33].

\section{Conclusions}

The agar gelled electrolyte allows to obtain repetitive and good quality EIS spectra. Some differences between the spectra of the liquid and gelled electrolyte have been observed, due to an increase in conductivity and a slight depolarization effect of the agar. For application on cultural heritage studies, these effects can usually be disregarded since the results are reproducible and allow obtaining comparative data of different substrates, patinas and coatings, and follow their evolution with time. In consequence, agar gel polymer electrolyte (GP-E) cell has proved to be an interesting alternative to carry out in-situ EIS tests on metallic cultural heritage, solving some of the difficulties of the experimental setups used by other authors. The information obtained by this technique can provide conservators-restorers quantitative data about the corrosion protection properties of patinas and coatings.

\section{Acknowledgements}

This work has been funded by project HAR2011-22402 and FPI grant BES-2012-052716 within the Plan Nacional de I+D+i 2008-2011 of Spanish Ministerio de Ciencia e Innovación. The authors also want to thank Marisa and Miguel Angel Codina for their invaluable help with the preparation of bronze coupons. 


\section{References}

[1] E. Cano, D. Lafuente, D.M. Bastidas, Use of EIS for the evaluation of the protective properties of coatings for metallic cultural heritage: A review, Journal of Solid State Electrochemistry, 14 (2010) 381-391.

[2] E. Cano, D.M. Bastidas, V. Argyropoulos, S. Fajardo, A. Siatou, J.M. Bastidas, C. Degrigny, Electrochemical characterization of organic coatings for protection of historic steel artefacts, Journal of Solid State Electrochemistry, 14 (2010) 453-463.

[3] R.d.P.B. Hernández, I.V. Aoki, B. Tribollet, H.G. de Melo, Electrochemical impedance spectroscopy investigation of the electrochemical behaviour of copper coated with artificial patina layers and submitted to wet and dry cycles, Electrochimica Acta, 56 (2011) 2801-2814.

[4] H. Otmacic Curkovic, T. Kosec, K. Marušić, A. Legat, An electrochemical impedance study of the corrosion protection of artificially formed patinas on recent bronze, Electrochimica Acta, 83 (2012) 28-39.

[5] T. Kosec, H.O. Ćurković, A. Legat, Investigation of the corrosion protection of chemically and electrochemically formed patinas on recent bronze, Electrochimica Acta, 56 (2010) 722 731.

[6] C. Chiavari, K. Rahmouni, H. Takenouti, S. Joiret, P. Vermaut, L. Robbiola, Composition and electrochemical properties of natural patinas of outdoor bronze monuments, Electrochimica Acta, 52 (2007) 7760-7769.

[7] P. Letardi, A. Beccaria, M. Marabelli, G. D'Ercoli, Application of electrochemical impedance mesurements as a tool for the characterization of the conservation and protection state of bronze works of art, in: J. Ashton, D. Hallam (Eds.) Metal 98. International Conference on Metal Conservation James \& James, Draguignan-Figanières (France), 1998, pp. 303-308.

[8] P. Letardi, R. Spiniello, Characterisation of bronze corrosion and protection by contactprobe electrochemical impedance measurements, in: I. MacLeod, J.M. Theile, C. Degrigny (Eds.) Metal 01. International Conference on Metal Conservation Western Australian Museum, Santiago (Chile), 2001, pp. 316-319.

[9] E. Angelini, S. Grassini, S. Corbellini, G.M. Ingo, T. De Caro, P. Plescia, C. Riccucci, A. Bianco, S. Agostini, Potentialities of XRF and EIS portable instruments for the characterisation of ancient artefacts, Applied Physics A: Materials Science and Processing, 83 (2006) 643-649.

[10] E. Angelini, S. Grassini, M. Parvis, F. Zucchi, An in situ investigation of the corrosion behaviour of a weathering steel work of art, Surface and Interface Analysis, 44 (2012) 942-946.

[11] S. Corbellini, M. Parvis, S. Grassini, Noninvasive solution for electrochemical impedance spectroscopy on metallic works of art, IEEE Transactions on Instrumentation and Measurement, 61 (2012) 1193-1200.

[12] A.H. England, T.L. Clare, Synthesis and characterization of flexible hydrogel electrodes for electrochemical impedance measurements of protective coatings on metal sculptures, Electroanalysis, 26 (2014) 1059-1067.

[13] V. Di Noto, S. Lavina, G.A. Giffin, E. Negro, B. Scrosati, Polymer electrolytes: Present, past and future, Electrochimica Acta, 57 (2011) 4-13. 
[14] M. Lahaye, C. Rochas, Chemical structure and physico-chemical properties of agar, Hydrobiologia, 221 (1991) 137-148.

[15] S. Arnott, A. Fulmer, W.E. Scott, I.C.M. Dea, R. Moorhouse, D.A. Rees, The agarose double helix and its function in agarose gel structure, Journal of Molecular Biology, 90 (1974) 269-284.

[16] E. Raphael, C.O. Avellaneda, B. Manzolli, A. Pawlicka, Agar-based films for application as polymer electrolytes, Electrochimica Acta, 55 (2010) 1455-1459.

[17] R. Leones, F. Sentanin, L.C. Rodrigues, I.M. Marrucho, J.M.S.S. Esperança, A. Pawlicka, M.M. Silva, Investigation of polymer electrolytes based on agar and ionic liquids, Express Polymer Letters, 6 (2012) 1007-1016.

[18] A.C. Nwanya, C.I. Amaechi, A.E. Udounwa, R.U. Osuji, M. Maaza, F.I. Ezema, Complex impedance and conductivity of agar-based ion-conducting polymer electrolytes, Applied Physics A: Materials Science and Processing, 119 (2015) 387-396.

[19] E. Cano, A. Crespo, D. Lafuente, B. Ramírez Barat, A novel gel polymer electrolyte cell for in-situ application of corrosion electrochemical techniques, Electrochemistry Communications, 41 (2014) 16-19.

[20] S.N. Inamdar, M.A. Bhat, S.K. Haram, Construction of $\mathrm{Ag} / \mathrm{AgCl}$ Reference Electrode from Used Felt-Tipped Pen Barrel for Undergraduate Laboratory, Journal of Chemical Education, 86 (2009) 355-356.

[21] E. Bernardi, C. Chiavari, C. Martini, L. Morselli, The atmospheric corrosion of quaternary bronzes: An evaluation of the dissolution rate of the alloying elements, Applied Physics A: Materials Science and Processing, 92 (2008) 83-89.

[22] P. Letardi, Electrochemical impedance measurements in the conservation of metals, in: D.C. Creagh, O.A. Bradley (Eds.) Radiation in Art and Archeometry, Elsevier Science B.V, Amsterdam, 2000, pp. 15-39.

[23] M.E. Orazem, N. Pébère, B. Tribollet, Enhanced graphical representation of electrochemical impedance data, Journal of the Electrochemical Society, 153 (2006) B129B136.

[24] J.B. Jorcin, M.E. Orazem, N. Pébère, B. Tribollet, CPE analysis by local electrochemical impedance spectroscopy, Electrochemical Impedance Spectroscopy Selection of Papers from the 6th International Symposium (EIS 2004), 51 (2006) 1473-1479.

[25] C.L. Alexander, B. Tribollet, M.E. Orazem, Contribution of Surface Distributions to Constant-Phase-Element (CPE) Behavior: 1. Influence of Roughness, Electrochimica Acta, 173 (2015) 416-424.

[26] P. Cördoba-Torres, T.J. Mesquita, R.P. Nogueira, Relationship between the origin of constant-phase element behavior in electrochemical impedance spectroscopy and electrode surface structure, Journal of Physical Chemistry C, 119 (2015) 4136-4147.

[27] R. De Levie, Electrochemical response of porous and rough electrodes, in: P. Delahay (Ed.) Advances in Electrochemistry and Electrochemical Engineering, Interscience Publishers, Inc, New York, 1967. 
[28] Y. Feng, W.K. Teo, K.S. Siow, K.L. Tan, A.K. Hsieh, The corrosion behaviour of copper in neutral tap water. Part I: Corrosion mechanisms, Corrosion Science, 38 (1996) 369-385.

[29] S.O. Lesmana, N. Febriana, F.E. Soetaredjo, J. Sunarso, S. Ismadji, Studies on potential applications of biomass for the separation of heavy metals from water and wastewater, Biochemical Engineering Journal, 44 (2009) 19-41.

[30] V.J.P. Vilar, C.M.S. Botelho, R.A.R. Boaventura, Modeling equilibrium and kinetics of metal uptake by algal biomass in continuous stirred and packed bed adsorbers, Adsorption, 13 (2007) 587-601.

[31] V.J.P. Vilar, C.M.S. Botelho, R.A.R. Boaventura, Copper removal by algae Gelidium, agar extraction algal waste and granulated algal waste: Kinetics and equilibrium, Bioresource Technology, 99 (2008) 750-762.

[32] A. Crespo, B. Ramírez Barat, D. Lafuente, S. Diaz, E. García, E. Cano, Non-destructive electrochemical evaluation of the patinas on the bronze sphinxes of the Museo Arqueológico Nacional in Madrid, Art'14. 11 th International Conference on non-destructive investigations and microanalysis for the diagnostics and conservation of cultural and environmental heritage.Madrid, 2014.

[33] B. Ramírez Barat, E. Cano Díaz, Evaluación in situ de recubrimientos protectores para patrimonio cultural metálico mediante espectroscopía de impedancia electroquímica, GEConservación, 8 (2015) in press. 